\title{
Introduction to Special Issue: Culturally Responsive School-Based Mental Health Interventions
}

\author{
Sara M. Castro-Olivo ${ }^{1}$
}

Published online: 5 July 2017

(C) California Association of School Psychologists 2017

The number of students (and families) that identified as culturally and linguistically diverse (CLD) in the USA, and around the world, continues to increase. Currently, in the USA, over $39 \%$ of the overall population identify as a racial/ ethnic minority (U.S. Census Bureau 2015). This diversity is even more prominent among school-aged populations; where half of the students enrolled in public schools identify as an ethnic/racial minority. Most specifically, 25\% of students enrolled in public schools identify as Hispanic; $16 \%$ as African American; 5\% as Asian; 50\% as white; and $4 \%$ as other (NCES 2016a). Although only 9.4\% (4.6 million students) are classified as English Language Learners (ELLs) at the national level in public schools (NCES 2016b), more than $20 \%$ of children report speaking a language other than English at home or are bilinguals (Federal Interagency Forum on Child and Family Statistics [FIFCFS] 2015). The percentage of students classified as ELLs is even higher in some states than the national average. For example, in California, 22.4\% of students enrolled in public schools receive English Language Development (ELD) services. Nevada, Texas, and New Mexico have at least 15\% of their student population receiving ELL services in addition to an even greater proportion of students who identify as an ethnic/ racial minority and/or speak a language other than English at home (NCES 2016b).

Although the percentage of CLD students in public schools continues to increase, many practitioners still struggle to identify evidence-based treatments (EBTs) that have shown to be effective, or even promising, for children who come from

Sara M. Castro-Olivo

s.castro-olivo@tamu.edu

1 Texas A\&M University, College Station, TX, USA diverse cultural and language backgrounds. Regardless of the cultural or racial background of the student populations served by public schools, schools have been identified as the perfect setting for implementing mental health prevention programs and identifying children and families in need of additional resources (The President's New Freedom Commission on Mental Health 2003). More and more schools are now adopting public health frameworks, where multi-tiered levels of support can help them better identify and treat children and adolescents in need of mental health services. These approaches could ultimately help reduce the extant disparities in mental health problems and treatments for culturally and linguistically (CLD) populations. However, much more research is needed to determine the practices that will yield the best, and lasting, results for these populations. Practitioners and researchers continue to ask questions such as: "Could CLD populations respond equally to EBTs developed for mainstream populations?" "What cultural factors, if any, need to be addressed in order for our school-based interventions to be culturally and socially valid for CLD?"

To this date, most existing evidence-based interventions have been developed with a mainstream population in mind, almost completely ignoring the impact culture, race, SES, and language proficiency could have on students' social-emotional, behavioral, and academic outcomes (Castro Olivo 2010). Previous research has shown that "culture" matters in the way children and adolescents develop, view the world, and incorporate themselves into a larger society (U.S. Department of Human Health Services 2001). Research has shown that CLD populations present higher prevalence of mental health problems and access to care when compared to whites (Alegria et al. 2008). Individuals with limited English language proficiency have been found to struggle more with mental health problems; as they are less likely to seek services (Bauer et al. 2010). In addition, retention and satisfaction of mental health 
treatment has been found to be lower for ethnic minority populations when compared to that of white clients (Castro et al. 2004; Fotuna et al. 2010). These disparities suggest that CLD populations are not only at higher risk for developing problems due to the many systemic challenges they face (i.e., lower SES, language barriers, discrimination), but also have less opportunities to access the care they need to bounce back and live fully productive and satisfying lives. Despite these findings, most evidence-based interventions used in schools or clinical settings to treat children with symptoms of depression, anxiety, or to just promote resiliency do not address the integration of cultural components into their procedures (Huey and Polo 2008). Research has shown that CLD individuals who are exposed to interventions that have been culturally adapted to explicitly meet their cultural reality report high levels of acceptance and satisfaction, in addition to improved outcomes (Hall et al. 2016; Smith et al. 2011). This type of research is still emerging for the youth population with only one meta-analysis to date indicating that current research on the effectiveness of culturally responsive intervention for youth is limited by the few studies that have focused, or fully described culturally responsive practices on their procedures, or due to small sample sizes (Huey and Polo 2008). In a systematic review of the extant literature on culturally grounded prevention programs (i.e., programs that were designed with a specific ethnic minority population in mind, but not an adaptation of an evidence-based treatment), Lauricella et al. (2016) found that of the 31 studies that employed culturally grounded programs reviewed, the majority is found to have been effective at improving outcomes for participants. Although this finding is promising, Lauricella and colleagues found that most of these prevention programs focused on preventing HIV, substance use, and early pregnancy. More research is still needed in regard to the effects of culturally responsive practices on prevention and intervention of mental health problems like depression and anxiety in school-based settings. Several researchers who have carefully adapted their interventions to meet the specific cultural realities of their target populations within school settings, and have followed a comprehensive framework like the one proposed by Guillermo Bernal and colleagues in 1995, have noted that these practices are effective, feasible within the regular school schedule, and socially valid among their participants (Castro-Olivo and Merrell 2012; Castro-Olivo 2014; Kramer and Castro-Olivo 2016; Preciado et al. 2009).

The purpose of this special issue is to increase the awareness of the need for more school-based research that employs culturally responsive practices. This special issue also serves as an outlet for researchers committed to addressing the unique mental health needs of CLB populations within school settings. Readers will find that authors of the included papers did an excellent job at describing the challenges CLD populations face and the many sociocultural factors researchers and practitioners must take into account when serving CLD students. This special issue is composed of six papers, two of which focus on providing overviews of the current literature and examples of models, practitioners can employ to make their practices more culturally responsive (i.e., Petterson, Villareal \& Castro; Goforth, Nichols, Stanick, Shindorf \& Holfer). In addition, four papers describe the results of studies (ranging from case studies to mix-method approaches) that examined the effectiveness of culturally responsive mental health and consultation practices with diverse populations within school settings (Falcon \& Mueller; Ijadi-Magsoodi, Marlotte, Garcia, Aralis, Lester \& Kataoka; Castro-Villraeal \& Rodriguez).

The first article by Dr. Petterson and colleagues highlights the need for making interventions culturally responsive for CLD students. They summarize the current literature on models that have been validated for making existing interventions more culturally responsive. They discussed how these models can be employed in school settings and the need for training programs to emphasize cultural competency in their programs as this is a key skill for future practitioners. Practitioners and researchers will find this article to be very informative and a great resource as they start planning cultural adaptations for their CLD populations.

Dr. Goforth and colleagues in their article titled: "SchoolBased Considerations for Supporting Arab American Youth's Mental Health," provide us with a detailed review illustrating the diversity among Arab American youth and the many challenges they face in our current society. In this article, the authors make suggestions for supporting Arab American youth through the implementation of school-wide multi-tiered levels of support. They discuss the many sociocultural factors practitioners must consider when assessing and intervening with Arab American youth at each level of the intervention tiers.

Drs. Fallon's and Muller's article illustrates the steps practitioners can take when working with CLD students with intense needs. Also following a multi-tiered level of support approach, they discuss how wraparound services can be made more cultural responsive to ensure cultural/social acceptability and sustainability of intervention. The authors report the procedures and results of a case study where a recent immigrant Puerto Rican family received wraparound services to best support the behavioral needs of a 13-year-old girl.

In their article titled "Culturally Responsive Adaptations in Evidence-Based Treatments: The Impact on Client Satisfaction," Dr. Jones and colleagues report the results of a study where clients' satisfaction was found to increase more rapidly when they received services from clinicians who had been trained on Multicultural Counseling Competencies (MCCs) versus those who received services from clinicians who provided regular cognitive behavioral therapy. Multicultural Counseling Competencies and ways that can be implementing in school settings are carefully described in this article. The authors make a strong argument for endorsing 
this approach with all students regardless of their ethnic/race background. Although more research is needed in this area, this article sheds light about the importance on MCCs and evaluating clients' satisfaction as an outcome variable when working with students with depression and anxiety.

Ijadi-Maghsoodi and colleagues in their manuscript: "Adapting and Implementing a School-Based ResilienceBuilding Curriculum Among Low-Income Racial and Ethnic Minority youth," present the procedures they follow to adapt the Resilience Classroom Curriculum in a predominantly ethnic/racial minority setting. They followed a communitypartnered participatory research approach (Jones and Wells 2007); where focus groups were conducted to evaluate the cultural alignment between the participating students' culture and the culturally adapted curriculum. In addition, the authors consulted with school administrators in regard to the measure and constructs to evaluate. Although the program was not adapted with one ethnic/racial group in mind, the authors considered students' SES and language background when adapting the lessons and examples of the program. The intervention consisted of nine modules that taught students emotion regulation communication, problem solving, goal setting, and managing stress reminders. Students, who participated in this pilot study, reported increased levels of empathy and problem solving at post intervention assessments. Students also reported high levels of social validity/acceptability of the program.

Consulting with teachers from diverse backgrounds who are working with culturally and linguistically diverse students should also be a priority in our field. Drs. Castro-Villarreal and Rodriguez in their article titled "Using Consulted-Centered Consultation with Teachers in a Contemporary School Setting to Inform Culturally Responsive Practice," discuss the results of their mixed methods study where they examined the perceptions, and experiences, of eight culturally diverse teachers, who received consultation from four school psychology graduate students. Participating teachers identified a collaborative approach to be more effective for them. They also identified the need for consultants to spend more time at their sites and work directly with the students in order to earn their trust. Although participating teachers in this study reported mixed levels of satisfaction with the consultation process, the data presented shows that the interventions the consultants supported were effective at improving students' outcomes. These findings suggest the need to increase the culturally responsive needs of both teachers and students to ensure higher levels of satisfaction and social acceptability from diverse teachers when consulting.

\section{Conclusion and Future Directions}

CLD populations are in greater need for school-based interventions that explicitly address their "cultural reality." School psychologists are in the perfect position for helping identify, prevent, and treat mental health issues in school settings. Practitioners and researchers will find the articles in this special issue to be stimulating as they present different models and explicit examples on how to make mental health and consultation practices more culturally responsive for students, their families, and teachers. I also hope this special issue will highlight the continued need for larger randomized control trials that evaluate the effectiveness of culturally responsive programs (either prevention or intervention) for CLD populations at risk for depression and anxiety. I am very grateful for the valuable contributions these authors have made to the field of school-based culturally responsive practices. We have a lot more work to be done, but I find it extremely refreshing and inspiring to see the level of commitment many of my colleagues and the field of school psychology have for supporting CLD students and families experiencing mental health difficulties.

\section{References}

Alegria, M., Chatter, P., Wells, K., Cao, Z., Chen, C., Takeuchi, D., Jackson, J., \& Meng, X. (2008). Disparity in depression treatment among racial and ethnic minority populations in the United States. Psychiatry, 59(11), 1264-1272.

Bauer, A. M., Chen, C., \& Alegria, M. (2010). English language proficiency and mental health services use among Latino and Asian Americans with mental disorders. Medical Care, 48(12), 10971104.

Castro Olivo, S. (2010). One size does not fit all: adapting SEL programs for use in our multicultural world. In K. W. Merrell \& B. A. Gueldner (Eds.), Social and Emotional Learning in the Classroom: Promoting Mental Health and Academic Success. New York: Guildford Publications Inc..

Castro-Olivo, S. (2014). The impact of a culturally adapted socialemotional learning program on ELL students' resiliency outcomes. School Psychology Quarterly, 29(4), 567-577. doi:10.1037/ spq0000055.

Castro-Olivo, S., \& Merrell, K. W. (2012). Validating cultural adaptations of a school-based social-emotional learning program for use with Latino immigrant adolescents. Advances in School Mental Health Promotion, 5(2), 78-92.

Castro, F. G., Barrera Jr, M., \& Martinez Jr, C. R. (2004). The cultural adaptation of prevention interventions: Resolving tensions between fidelity and fit. Prevention Science, 5(1), 41-45.

Federal Interagency Forum on Child and Family Statistics. (2015). America's children: Key national indicators of well-being, 2015. Washington, DC: U.S. Government Printing Office. Retrieved from:http://www.childstats.gov/pdf/ac2015/ac_15.pdf

Fotuna, L. R., Alegria, M., \& Gao, S. (2010). Retention in depression treatment among ethnic and racial minority groups in the United States. Depression and Anxiety, 27, 485-494.

Hall, G. C. N., Ibaraki, A. Y., Huang, E. R., Marti, C. N., \& Stice, E. (2016). A meta-analysis, of cultural adaptations of psychological interventions. Behavior Therapy, 47, 993-1014.

Huey, S. J., \& Polo, A. J. (2008). Evidence-based psychosocial treatments for ethnic minority youth. Journal of Clinical Child \& Adolescent Psychology, 37(1), 262-301. 
Jones, L., \& Wells, K. (2007). Strategies for academic and clinician engagement in community participatory partnered research. Jama, 297 (4), 407-410.

Kramer, C., \& Castro-Olivo, S. (2016). Social-emotional learning intervention outcomes of culturally diverse high school students. Contemporary School Psychology Journal, 20(2), 118-129.

Lauricella, M., Valdez, J. K., Okamoto, S. K., Helm, S., \& Zaremba, C. (2016). Culturally grounded prevention for minority youth populations: A systematic review of the literature. Primary Prevention, 37, $11-32$.

National Center for Educational Statistics (20016a). Status and trends in the education of racial and ethnic groups 2016. Retrieved from: https://nces.ed.gov/pubs2016/2016007.pdf.

National Center for Educational Statistics (2016b). English language learners in public schools. Retrieved from: https://nces.ed.gov/ programs/coe/indicator_cgf.asp.

Preciado, J.A., Horner, R.H., \& Baker, S.K. (2009). Using a functionbased approach to decrease problem behaviors and increase academic engagement for Latino English language learners. The Journal of Special Education, 42, 227-240.

Smith, T. B., Rodriguez, M. D., \& Bernal, G. (2011). Culture. Journal of Clinical Psychology, 67, 166-175. doi:10.1002/jclp.20757.

The Presidents' New Freedom Commission on Mental Health (2003). Achieving the promise: Transforming mental health care in
America. Retrieved from: http://govinfo.library.unt.edu/ mentalhealthcommission/reports/FinalReport/downloads/ FinalReport.pdf.

U.S. Department of Health and Human Services. (2001). Mental health: culture, race, and ethnicity - a supplement to mental health: a report of the surgeon general. Rockville: U.S. Department of Health and Human Services, Public Health Service, Office of the Surgeon General.

U.S. Census Bureau. (2015). Census Bureau reports at least 350 languages spoken in U.S. homes. Retrieved from: https://www. census.gov/newsroom/press-releases/2015/cb15-185.html

Sara Castro-Olivo $\mathrm{PhD}, \mathrm{NCSP}$ is an associate professor in the School Psychology Program at Texas A\&M University. Her research focuses on the development and validation of culturally responsive social-emotional and behavioral interventions for Latino ELL youth and families. She is also interested in studying the relationship between socio-cultural factors and academic performance of ELLs and refugee children. She has authored numerous chapters and research articles. She has many years of experience working with Spanish-speaking youth and families. 